\title{
Infected cardiac hydatid cyst
}

\author{
M Ceviz, N Becit, H Koçak
}

\begin{abstract}
A 24 year old woman presented with chest pain and palpitation. The presence of a semisolid mass-an echinococcal cyst or tumour-in the left ventricular apex was diagnosed by echocardiography, computed tomography, and magnetic resonance imaging. The infected cyst was seen at surgery. The cyst was removed successfully by using cardiopulmonary bypass with cross clamp.

(Heart 2001;86:e13)
\end{abstract}

Keywords: cardiac hydatid cyst; infected cardiac hydatid cyst

Cardiac involvement of hydatid disease is uncommon, occurring in less than $2 \%$ of cases. ${ }^{1}$ When cardiac involvement is present, the cysts usually are intramyocardial in the interventricular septum of the left ventricular free wall. The right ventricle or atrium may be involved. ${ }^{1}$ We present a case of left ventricular infected hydatid cyst.

\section{Case report}

A 24 year old woman was admitted to our hospital with chest pain and palpitation for the previous two years. Negative $T$ waves were seen on the anterior and inferior electrocardiographic leads. A transthoracic echocardiogram showed a left apical solid mass. There was a cystic structure in the mass. Computed tomography (CT) (fig 1) and magnetic resonance imaging showed a semisolid mass, $5 \times 6 \mathrm{~cm}$ in size, in the apex of left ventricle. An echinococcal haemagglutination test was positive with a

Department of Cardiovascular Surgery, Medical Faculty, Atatürk University, 25240 Erzurum, Turkey M Ceviz

N Becit

H Koçak

Correspondence to: Dr Ceviz ceviz@tr.net

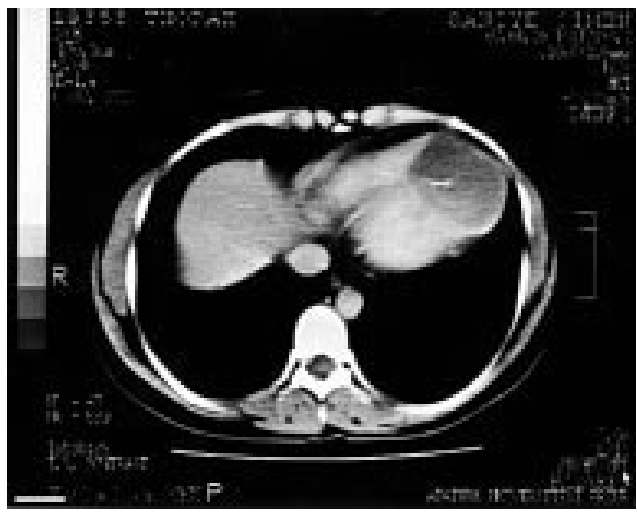

Figure 1 Preoperative CT scan showing a semisolid mass in the left ventricular apex. titre of 80. Coronary angiographic study showed normal vessels.

The patient underwent elective surgery on 17 May 2000 through a median sternotomy. Cardiopulmonary bypass was initiated using bicaval cannulae and an arterial cannula positioned in the ascending aorta. Antegrade crystalloid cardioplegia was used. The mass was seen clearly in the muscle of the left ventricular apex (fig 2A). The area in which the cyst was situated was isolated from the rest of the heart and the pericardial cavity with gauze packs. An incision was made to this area. The cystic cavity was opened and pus content of the cyst was aspirated with a transparent large bore cannula (fig 2B). Ruptured cyst membranes were removed. The cavity had no communication with the ventricular cavity (fig 2C). The cyst was irrigated with a polyvinyl pyrolidone solution. After partial resection, the cyst cavity was closed with the capitonage technique and primer suturing without felt (fig 2D). The patient tolerated the procedure and her postoperative course was uneventful. Microbiological study showed methicillin sensitive staphylococcus that were coagulase positive. Imipenem and cefazolin sodium were given in the postoperative period for seven days. The patient was discharged on the ninth day. Echocardiography found no further accumulation during the second, sixth, and 11th postoperative months. Histopathological examination showed abscess material. She did well during the first year of follow up. CT was not performed because she was pregnant.

\section{Discussion}

The cestode tapeworm, Echinococcus granulosis, causes hydatic disease of the heart. The life cycle of this parasite and the natural course of hydatid disease are well described. ${ }^{2}$

The diagnosis of cardiac echinococcocis can be difficult and must be suspected in any patient from sheep farming areas with a cystic tumour of the heart. ${ }^{3}$ The clinical picture may vary from asymptomatic to congestive heart failure. ${ }^{4}$ Echocardiography is accurate for diagnosis and determining the number, location, and size of the cysts. ${ }^{1}$ CT and magnetic resonance imaging are used for diagnosis. Coronary studies should be done in symptomatic patients or when electrocardiographic abnormalities are present as in our case.

In contrast with the typical cystic image, some patients show solid masses on the 

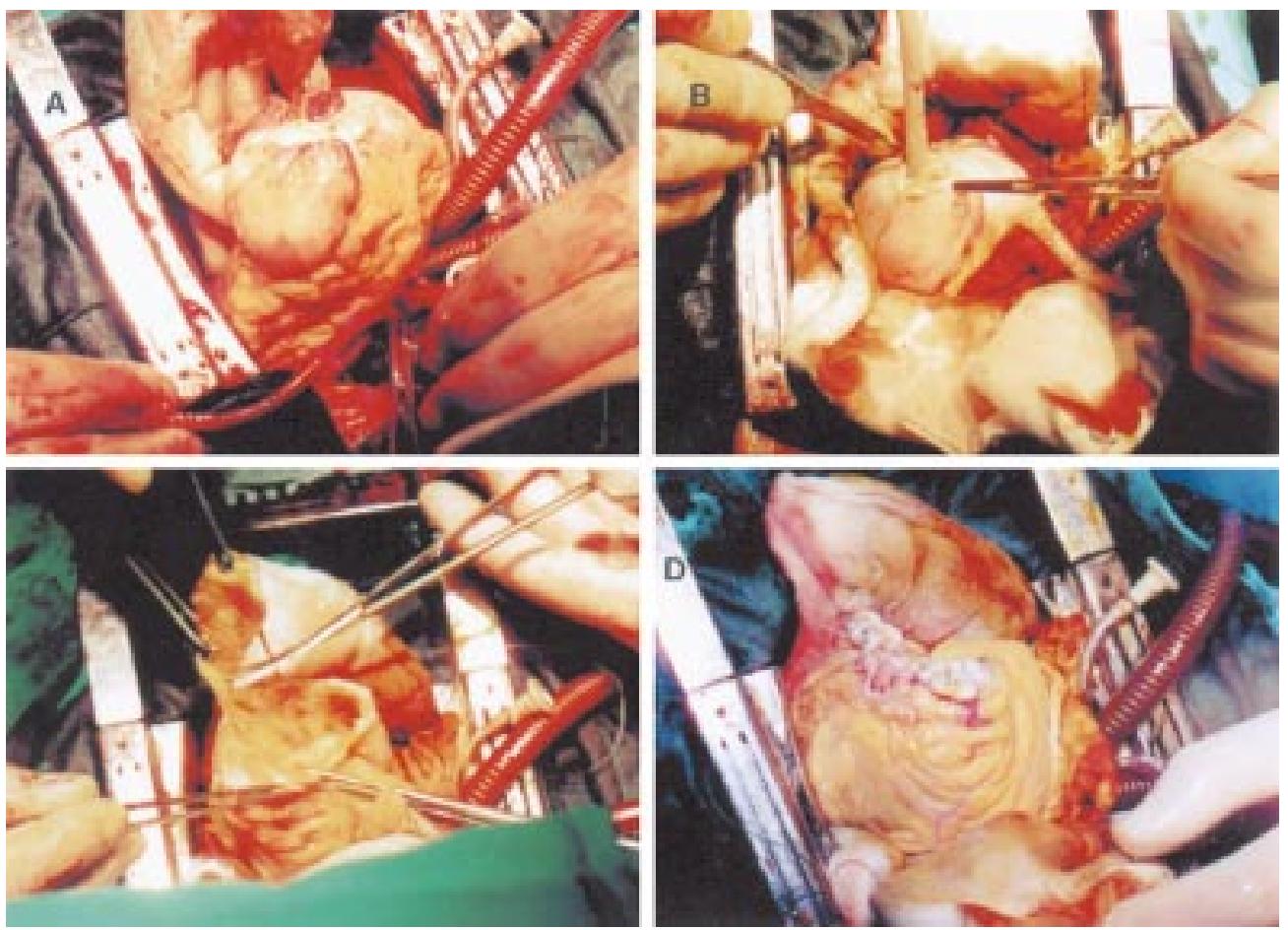

Figure 2 Intraoperative view. (A) The mass is seen clearly in the muscle of the left ventricular apex. (B) The cystic cavity was opened and pus inside the cyst was aspirated with a transparent large bore cannula. Ruptured cystic membranes were removed. (C) Partial resection. (D) The cystic cavity was closed with the capitonage technique and primer suturing without felt.

echocardiogram, which makes these cysts more difficult to differentiate from other primary or secondary heart tumours. ${ }^{1}$ In the series reported by Oliver and colleagues, ${ }^{1}$ pathological examination of the four cysts that appeared as solid masses on echocardiography showed that they had undergone a degenerative process that replaced the normal contents of the cyst with necrotic material containing membrane residues surrounded by inflammatory tissue containing a large number of giant cells. These authors, however, did not provide an explanation for the infection. In our case, there was solid imaging caused by the cystic infection.

Surgery is the treatment of choice for this condition. ${ }^{3}$ Intraoperative surface echocardiography gives a definitive diagnosis and can help in planning the operation by aiding identification of the cannulation site and in making decisions such as whether to use cardiopulmonary bypass and whether to perform the surgery on a beating heart or under cross clamp. ${ }^{5}$ We did not use surface echocardiography. We performed the operation under cross clamp because we could not distinguish the tumour from the cyst and we did not know whether it communicated with the ventricular cavity.

Four patients have undergone surgery in our department, ${ }^{6}$ the last for infected hydatid cyst. We found no other cases of infected cardiac hydatid cyst in the literature.

1 Oliver JM, Sotillo JF, Dominguez FJ, et al. Two-dimensional echocardiographic features of echinococcosis of the heart and great blood vessels: clinical and surgical implications. Circulation 1988;78:327-37.

2 Ameli M, Maborhan HA, Nauraii SS. Surgical treatment of hydatid cysts of the heart: report of six cases. $\mathcal{F}$ Thorac Cardivasc Surg 1989:98:892-901.

3 Rassouw GJ, Knott-Craig CJ, Erasmus PA. Cardiac echinococcosis: cyst removal in a beating heart. Ann Thorac Surg 1992;53:328-9.

4 Heyat J, Mokhtari H, Hajallioo J, et al. Surgical treatment of echinococcal cyst of the heart. $\mathcal{F}$ Thorac Cardiovasc Surg 1971;61:755-64.

5 Birincioğlu CL, Bardakcı H, Küçüker ŞA, et al. A clinical dilemma: cardiac and pericardiac echinococcosis. Ann Thorac Surg 1998;68;1290-4.

6 Yekeler I, Koçak H, Aydın NE, et al. A case of cardiac hydatid cyst localized in the lungs bilaterally and on anterior wall of right ventricle. Thorac Cardivasc Surg 1993; 41:261-3.

7 Özyazıcıoğlu A, Koçak H, Becit N. Cardiac and pericardiac echinococcosis. Ann Thorac Surg 2000;70:1002-4. 